TRANSACTIONS OF THE

AMERICAN MATHEMATICAL SOCIETY

Volume 361, Number 6, June 2009, Pages 3109-3123

S 0002-9947(08)04692-8

Article electronically published on December 30, 2008

\title{
STURMIAN AND SPECTRAL THEORY FOR DISCRETE SYMPLECTIC SYSTEMS
}

\author{
MARTIN BOHNER, ONDŘEJ DOŠLÝ, AND WERNER KRATZ
}

\begin{abstract}
We consider $2 n \times 2 n$ symplectic difference systems together with associated discrete quadratic functionals and eigenvalue problems. We establish Sturmian type comparison theorems for the numbers of focal points of conjoined bases of a pair of symplectic systems. Then, using this comparison result, we show that the numbers of focal points of two conjoined bases of one symplectic system differ by at most $n$. In the last part of the paper we prove the Rayleigh principle for symplectic eigenvalue problems and we show that finite eigenvectors of such eigenvalue problems form a complete orthogonal basis in the space of admissible sequences.
\end{abstract}

\section{INTRODUCTION AND MAIN RESULTS}

In this paper we deal with oscillation properties of symplectic difference systems

$$
z_{k+1}=\mathcal{S}_{k} z_{k}, \quad k \in\{0, \ldots, N\}
$$

where the matrices $\mathcal{S}_{k}$ are symplectic, i.e.,

$$
\mathcal{S}^{T} \mathcal{J} \mathcal{S}=\mathcal{J}, \quad \text { where } \quad \mathcal{J}=\left(\begin{array}{cc}
0 & I \\
-I & 0
\end{array}\right) .
$$

(Note that here and in the entire paper we use the convention that an equation written as $A=B$ means that $A_{k}=B_{k}$ for all $k \in\{0, \ldots, N\}$.) The system (S) is a natural discrete counterpart of the linear Hamiltonian differential system

$$
z^{\prime}=\mathcal{H}(t) z, \quad \text { where } \quad \mathcal{J} \mathcal{H}(t)+\mathcal{H}^{T}(t) \mathcal{J}=0, \quad t \in[a, b],
$$

whose oscillation theory is deeply developed; see, e.g., [22, 23, 21, 17]. Discrete symplectic systems play a key role in the numerical methods for solving Hamiltonian systems, since they "... present a proper way, i.e., the Hamiltonian way, for computing the Hamiltonian dynamics" [14, page 18]. Also, these systems are closely related to discrete quadratic functionals which arise as the second-order nonlinear problems in the discrete calculus of variations and optimal control theory; see, e.g., [15, 16, 24, 25, 19, 13, 26, 2,

Received by the editors June 20, 2007.

2000 Mathematics Subject Classification. Primary 39A12, 39A13, 34B24, 49K99.

Key words and phrases. Discrete symplectic system, discrete quadratic functional, Sturmian separation result, Sturmian comparison result, Rayleigh principle, extended Picone identity.

(C)2008 American Mathematical Society Reverts to public domain 28 years from publication 
If in $(\underline{\mathrm{S}})$ and $(\underline{\mathrm{H}})$ we write

$$
z=\left(\begin{array}{l}
x \\
u
\end{array}\right), \quad \mathcal{S}=\left(\begin{array}{cc}
\mathcal{A} & \mathcal{B} \\
\mathcal{C} & \mathcal{D}
\end{array}\right), \quad \mathcal{H}=\left(\begin{array}{cc}
A & B \\
C & -A^{T}
\end{array}\right)
$$

with $x, u \in \mathbb{R}^{n}$ and $\mathcal{A}, \mathcal{B}, \mathcal{C}, \mathcal{D}, A, B, C \in \mathbb{R}^{n \times n}$, then these systems can be rewritten in the forms

$$
x_{k+1}=\mathcal{A}_{k} x_{k}+\mathcal{B}_{k} u_{k}, \quad u_{k+1}=\mathcal{C}_{k} x_{k}+\mathcal{D}_{k} u_{k}, \quad k \in\{0, \ldots, N\},
$$

and

$$
x^{\prime}=A(t) x+B(t) u, \quad u^{\prime}=C(t) x-A^{T}(t) u, \quad t \in[a, b],
$$

respectively. A matrix solution $Z=(X, U)$ of $(\underline{S})$, where $X_{k}$ and $U_{k}$ are $n \times n$ matrices, is said to be a conjoined basis of $(\underline{S})$ if the matrices $X_{k}^{T} U_{k}$ are symmetric and $\operatorname{rank}\left(\begin{array}{c}X_{k} \\ U_{k}\end{array}\right)=n$ for all $k \in\{0, \ldots, N\}$. A conjoined basis of $(\mathbf{H})$ is defined in a similar way. Let $(X, U)$ and $(\tilde{X}, \tilde{U})$ be two conjoined bases of $(\underline{H})$ and denote by $m$ and $\tilde{m}$ the number of points in $[a, b]$ satisfying $\operatorname{det} X(t)=0$ and $\operatorname{det} \tilde{X}(t)=0$, respectively (the so-called focal points of $(X, U)$ and $(\tilde{X}, \tilde{U}))$. The basic statement of oscillation theory of $(\overline{\mathrm{H}})$ (the so-called Sturmian separation theorem for $(\overline{\mathrm{H}})$ ) states that $|m-\tilde{m}| \leq n$ [22, Chapter VII, Corollary 1 of Theorem 7.9 on page 366]. Of course, if $(\mathrm{H})$ corresponds to a second-order Sturm-Liouville equation, e.g.,

$$
\left(r(t) x^{\prime}\right)^{\prime}+p(t) x=0, \quad t \in[a, b],
$$

then this statement reduces to the classical Sturmian theorem about separation of zeros of linearly independent solutions of (SL).

The aim of this paper is, among others, to establish the analogue for conjoined bases of the discrete system $(\underline{S})$. To formulate this result, we will use the following notation and concepts. For a real and symmetric matrix $P$ we write $P \geq 0$ if $P$ is nonnegative definite, and ind $P$ denotes the index of $P$, i.e., the number of negative eigenvalues (including multiplicities) of $P$. By $\operatorname{Ker} M, \operatorname{Im} M, \operatorname{rank} M$, $M^{T}$, and $M^{-1}$ we denote the kernel, image, rank, transpose, and inverse of a matrix $M$, respectively. The notion of the Moore-Penrose inverse is fundamental in understanding the idea of multiplicity of a focal point as explained below, and therefore we will spend a little time discussing its definition and basic properties: For an $m \times n$-matrix $M$, there exists a unique $n \times m$-matrix $N$ satisfying $N M N=N$ and $M N M=M$ such that both $N M$ and $M N$ are symmetric (see [1, Section 2.8], [3. Theorem 1.5], [5, Appendix]). This matrix $N$ is called the Moore-Penrose inverse of $M$ and is denoted by $M^{\dagger}$. It can be explicitly given (see [1, Lemma 2.8.3], [17, Remark 3.3.2]) by

$$
M^{\dagger}=\lim _{t \rightarrow 0^{+}}\left\{\left(M^{T} M+t I\right)^{-1} M^{T}\right\}=\lim _{t \rightarrow 0^{+}}\left\{M^{T}\left(M M^{T}+t I\right)^{-1}\right\} ;
$$

in particular, these limits always exist, and we have

$$
\left(M^{T}\right)^{\dagger}=\left(M^{\dagger}\right)^{T}, \quad\left(M^{\dagger}\right)^{\dagger}=M, \quad \text { and } \quad \operatorname{Ker}\left(M^{\dagger}\right)^{T}=\operatorname{Ker} M .
$$

The main "reason" for the appearance of Moore-Penrose inverses in our theory is that for two matrices $V$ and $W$, the following equivalences hold (see [1, Lemma 2.8.6], [4, Lemma 4], [5, Lemma A5], [6, Remark 2 (ii) and (iii)]):

$$
\operatorname{Ker} V \subset \operatorname{Ker} W \quad \Longleftrightarrow W=W V^{\dagger} V \quad \Longleftrightarrow \quad W^{\dagger}=V^{\dagger} V W^{\dagger} \text {. }
$$


For a conjoined basis $(X, U)$ of $(\underline{S})$, the following matrices were introduced in [18:

$$
\left\{\begin{array}{l}
M_{k}=\left(I-X_{k+1} X_{k+1}^{\dagger}\right) \mathcal{B}_{k} \\
T_{k}=I-M_{k}^{\dagger} M_{k} \\
P_{k}=T_{k}^{T} X_{k} X_{k+1}^{\dagger} \mathcal{B}_{k} T_{k}
\end{array}\right.
$$

for $k \in\{0, \ldots, N\}$. Then obviously $M_{k} T_{k}=0$, and it can be shown (see, e.g., [18]) that the matrix $P_{k}$ is symmetric.

We say that a conjoined basis $(X, U)$ has no focal point [6, 8] in the interval $(k, k+1]$ if

$$
\operatorname{Ker} X_{k+1} \subseteq \operatorname{Ker} X_{k} \quad \text { and } \quad X_{k} X_{k+1}^{\dagger} \mathcal{B}_{k} \geq 0
$$

holds. Note that if the first condition in (1.2) holds, then the matrix $X_{k} X_{k+1}^{\dagger} \mathcal{B}_{k}$ is really symmetric (see [8]), and it equals the matrix $P_{k}$ given by (1.1) since $T_{k}=I$ in this case (see [18]). The multiplicity [18] of a focal point in the interval $(k, k+1]$ is defined as the number

$$
\operatorname{rank} M_{k}+\text { ind } P_{k} \text {. }
$$

Throughout this paper, focal points of any conjoined basis are counted, including their multiplicities.

Now we can formulate the Sturmian type separation theorem for conjoined bases of $(\underline{S})$, which is a discrete version of the above mentioned separation theorem for (H).

Theorem 1.1. The difference between the numbers of focal points in $(0, N+1]$ of any two conjoined bases of $(\underline{S})$ is at most $n$.

A natural extension of the Sturmian separation theorem is the Sturmian comparison theorem which compares the number of zeros of solutions of two equations $(\underline{\mathrm{SL}})$ or systems $(\mathrm{H})$. Together with $(\underline{\mathrm{S}})$, we consider the system

$$
z_{k+1}={\underset{\sim}{S}}_{k} z_{k}, \quad k \in\{0, \ldots, N\}, \quad \text { where } \quad \underset{\mathcal{S}}{\mathcal{S}}=\left(\begin{array}{cc}
\underset{\mathcal{A}}{\sim} & \mathcal{B} \\
\underset{\sim}{\mathcal{C}} & \mathcal{D}
\end{array}\right)
$$

and recall that the conjoined basis $Z=(X, U)$ of $(\underline{S})$ (and similarly for (ST) ) given by the initial condition $X_{0}=0, U_{0}=I$ is called the principal solution of (S) at 0 .

Theorem 1.2. Define the $2 n \times 2 n$-matrices

$$
\mathcal{G}:=\left(\begin{array}{cc}
\mathcal{A}^{T} \mathcal{B B}^{\dagger} \mathcal{D} \mathcal{B}^{\dagger} \mathcal{A}-\mathcal{A}^{T} \mathcal{C} & \mathcal{C}^{T}-\mathcal{A}^{T} \mathcal{B B}^{\dagger} \mathcal{D} \mathcal{B}^{\dagger} \\
\mathcal{C}-\left(\mathcal{B}^{\dagger}\right)^{T} \mathcal{D}^{T} \mathcal{B} \mathcal{B}^{\dagger} \mathcal{A} & \mathcal{B B}^{\dagger} \mathcal{D} \mathcal{B}^{\dagger}
\end{array}\right)
$$

and

and suppose

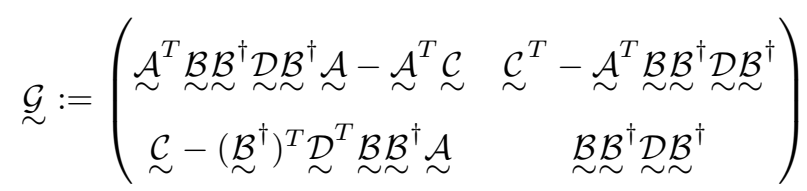

$$
\mathcal{G} \geq \underset{\mathcal{G}}{\sim} \quad \text { and } \quad \operatorname{Im}(\mathcal{A}-\mathcal{\mathcal { A }} \quad \mathcal{B}) \subset \operatorname{Im} \underset{\sim}{\mathcal{B}} .
$$


If the principal solution of (S) has $m$ focal points in $(0, N+1]$, then any conjoined basis of (S) has at most $m \stackrel{\sim}{+} n$ focal points in $(0, N+1]$.

Theorem 1.3. Suppose that (1.4) holds. If the principal solution of (S) has $m$ focal points in $(0, N+1]$, then any conjoined basis of (S) has at least $m$ focal points in $(0, N+1]$.

The proofs of these theorems are given in Sections 2 and 3 . These proofs are based on various results concerning eigenvalue problems associated with $(\underline{S})$ in the form

(E)

$$
\left\{\begin{array}{l}
x_{k+1}=\mathcal{A}_{k} x_{k}+\mathcal{B}_{k} u_{k}, \quad u_{k+1}=\mathcal{C}_{k} x_{k}+\mathcal{D}_{k} u_{k}-\lambda x_{k+1}, \quad k \in\{0, \ldots, N\}, \\
x_{0}=x_{N+1}=0 .
\end{array}\right.
$$

The eigenvalue problem (E) (including some preparatory material) is treated in Section 4 of this paper. In particular, attention is focussed on the Rayleigh principle for the discrete quadratic functional associated with $(\mathrm{E})$ and on the completeness of finite eigenvectors in the space of the so-called admissible sequences. Section 4 also contains some technical statements used in the proofs of the results of our paper.

\section{StURMIAN COMPARISON RESULTS}

An important role in the proof of Theorems 1.2 and 1.3 is played by the associated discrete quadratic functional

$$
\mathcal{F}(z)=\sum_{k=0}^{N}\left\{x_{k}^{T} \mathcal{A}_{k}^{T} \mathcal{C}_{k} x_{k}+2 x_{k}^{T} \mathcal{C}_{k}^{T} \mathcal{B}_{k} u_{k}+u_{k}^{T} \mathcal{B}_{k}^{T} \mathcal{D}_{k} u_{k}\right\} \quad \text { for } \quad z=\left(\begin{array}{l}
x \\
u
\end{array}\right) .
$$

A pair of $n$-dimensional sequences $z=\left\{z_{k}\right\}_{k=0}^{N+1}=\left\{\left(x_{k}, u_{k}\right)\right\}_{k=0}^{N+1}$ is said to be admissible for $\mathcal{F}$ provided it satisfies the first equation in $(\underline{S})$, i.e., the so-called equation of motion $x_{k+1}=\mathcal{A}_{k} x_{k}+\mathcal{B}_{k} u_{k}$ for all $k \in\{0, \ldots, N\}$.

Now we recall some concepts and statements associated with the eigenvalue problem (E). This eigenvalue problem is treated in detail in Section 4, but some results we need to present already now. A number $\lambda \in \mathbb{R}$ is called a finite eigenvalue of (E) if there exists a solution $z=(x, u)$ of (E) such that $x_{0}=0=x_{N+1}$ and $x=\left\{x_{k}\right\}_{k=0}^{N+1} \neq 0$, and then $z$ is called a finite eigenvector corresponding to $\lambda$. Let $(\tilde{X}, \tilde{U})=(\tilde{X}(\lambda), \tilde{U}(\lambda))$ be the principal solution of the symplectic system in (E) and denote by $\tilde{n}_{1}(\lambda)$ the number of focal points of $(\tilde{X}, \tilde{U})$ in $(0, N+1]$. Then by (4.8) given in Section 4 ,

$$
\tilde{n}_{1}(\lambda)=n_{2}(\lambda),
$$

where $n_{2}(\lambda)$ denotes the number of finite eigenvalues of (E) (counting multiplicities; see Definition 4.1 for details) which are less than or equal to $\lambda$.

The results in this section extend [16, Theorem 7.1], where the statements are proved (using a different technique than here, namely the Riccati technique) in the special case $m=0$ (see also [7, Theorem 2]). Here we also use the following lemma from [16, Formula (7.1)] (see also [20, Lemma 1.32]), which can be proved by a direct computation. 
Lemma 2.1. If $z=(x, u)$ is admissible and $\mathcal{G}$ is given by (1.3), then

$$
\mathcal{F}(z)=\sum_{k=0}^{N}\left(\begin{array}{c}
x_{k} \\
x_{k+1}
\end{array}\right)^{T} \mathcal{G}_{k}\left(\begin{array}{c}
x_{k} \\
x_{k+1}
\end{array}\right) .
$$

Proof of Theorem 1.2. Let $Z=(X, U)$ be any conjoined basis of $(\underline{S})$. Suppose that $Z$ has $p$ focal points in $(0, N+1]$. Then, corresponding to each focal point, we can construct $z^{[\nu]}=\left(x^{[\nu]}, u^{[\nu]}\right), 1 \leq \nu \leq p$, as in [11, (12) and (13) on page 338] such that

$$
x^{[\nu]} \quad \text { is admissible and } \quad x_{N+1}^{[\nu]}=0 \quad \text { for all } \quad 1 \leq \nu \leq p .
$$

Furthermore, since the principal solution $\underset{\sim}{Z}=(\underset{\sim}{X} \underset{\sim}{U})$ of $(\underset{\sim}{S})$ has $m$ focal points in $(0, N+1]$, by (2.1) with $\lambda=0$, applied to the eigenvalue problem

$$
\left\{\begin{array}{l}
x_{k+1}=\underset{\sim}{A_{k}} x_{k}+\underset{\sim}{\mathbb{Z}_{k}} u_{k}, \quad u_{k+1}=\underset{\sim}{C_{k}} x_{k}+\underset{\sim}{D_{k}} u_{k}-\lambda x_{k+1}, \quad k \in\{0, \ldots, N\}, \\
x_{0}=x_{N+1}=0,
\end{array}\right.
$$

this eigenvalue problem has $m$ nonpositive finite eigenvalues $\lambda_{\mu}, 1 \leq \mu \leq m$, with corresponding orthonormal finite eigenvectors $\underset{\sim}{z^{(\mu)}}=\left(\underset{\sim}{x^{(\mu)}},{\underset{\sim}{\sim}}^{(\mu)}\right), 1 \leq \mu \leq m$. Moreover, by Theorem 4.6 given in Section $4, \mathcal{F}(z) \geq \underset{\sim}{\mathcal{F}}(z)>0$ for $z=(x, u)$ satisfying

$$
z \perp z^{(\mu)}, \quad \text { i.e., } \quad\left\langle z, z^{(\mu)}\right\rangle:=\sum_{k=0}^{N} x_{k+1}^{T} \underset{\sim}{x_{k+1}^{(\mu)}}=0,1 \leq \mu \leq m, \quad x \neq 0 .
$$

Now suppose that $p>m+n$. Then there exists a nontrivial linear combination

$$
\sum_{\nu=1}^{p} c_{\nu}\left(\begin{array}{c}
\left\langle z^{[\nu]}, z^{(1)}\right\rangle \\
\left\langle z^{[\nu]}, z^{(2)}\right\rangle \\
\vdots \\
\left\langle z^{[\nu]}, z^{(m)}\right\rangle \\
x_{0}^{[\nu]}
\end{array}\right)=0 .
$$

Define

$$
z=(x, u)=\sum_{\nu=1}^{p} c_{\nu} z^{[\nu]} .
$$

By construction, $x_{N+1}=0$ and $x$ is admissible, as it is of the same form as in [11, (15) on page 339]. Moreover, $\sum_{\nu=1}^{p} c_{\nu} x_{0}^{[\nu]}=0$ implies $x_{0}=0$, and we also have

$$
0=\sum_{\nu=1}^{p} c_{\nu}\left\langle z^{[\nu]}, z^{(\mu)}\right\rangle=\left\langle z, z^{(\mu)}\right\rangle \quad \text { for all } \quad 1 \leq \mu \leq m,
$$

so $z \perp \tilde{z}^{(\mu)}$ for all $1 \leq \mu \leq m$. As in [11, Proof of Theorem 1 on page 339] we have $x \neq 0$ (the $x$ there was of the same form, and the only property that was used there was that not all $c_{\nu}=0$, which is guaranteed in our current setting) and $\mathcal{F}(z) \leq 0$. From the second condition in (1.4) it follows that there exists $\underset{\sim}{\sim}=\{\underset{\sim}{\sim}\}_{k=0}^{N}$ such 
that $x_{k+1}=\mathcal{A}_{k} x_{k}+\mathcal{B}_{k} \underset{\sim}{\sim}{ }_{k}$ for all $k \in\{0, \ldots, N\}$, and hence $\underset{\sim}{z}=(x, \underset{\sim}{u})$ is admissible for $\mathcal{F}$, and by applying Lemma 2.1 twice and coupled with the first condition in (1.4), we find

$$
\mathcal{F}(z)=\sum_{k=0}^{N}\left(\begin{array}{c}
x_{k} \\
x_{k+1}
\end{array}\right)^{T}{\underset{\sim}{\sim}}_{k}\left(\begin{array}{c}
x_{k} \\
x_{k+1}
\end{array}\right) \leq \sum_{k=0}^{N}\left(\begin{array}{c}
x_{k} \\
x_{k+1}
\end{array}\right)^{T} \mathcal{G}_{k}\left(\begin{array}{c}
x_{k} \\
x_{k+1}
\end{array}\right)=\mathcal{F}(z) \leq 0 .
$$

Hence we have found an admissible $z=(x, u)$ with $x \neq 0, x_{0}=x_{N+1}=0, z \perp{\underset{\sim}{\sim}}^{(\mu)}$ for all $1 \leq \mu \leq m$, and $\underset{\sim}{\mathcal{F}}(z) \leq 0$, contradicting the Rayleigh principle, Theorem 4.6 as stated and proved in Section 4, by which $\underset{\sim}{\mathcal{F}}(z)>0$ for all admissible $z$ with $x_{0}=x_{N+1}=0, x \neq 0$, and $z \perp{\underset{\sim}{z}}^{(\mu)}$ for all $1 \leq \mu \leq m$.

Proof of Theorem 1.3. We consider (E). Let $(X, U)=(X(\lambda), U(\lambda))$ be the principal solution of the symplectic system in $(\underline{\mathrm{E}})$ and let $\underset{\sim}{X}, \underset{\sim}{U})=(\underset{\sim}{X}(\lambda), \underset{\sim}{U}(\lambda))$ be any conjoined basis of the symplectic system in (2.2) such that $\underset{\sim}{X_{0}}(\lambda) \equiv \underset{\sim}{X_{0}}$ and $\underset{\sim}{U_{0}}(\lambda) \equiv \underset{\sim}{U_{0}}$ are constant. Let $m=n_{1}(\lambda)$ and $p=p(\lambda)$ be the numbers of focal points of $(X, U)$ and $\underset{\sim}{X}, \underset{\sim}{U})$ in $(0, N+1]$, respectively. Then the assertion of Theorem 1.3 says

$$
n_{1}(0) \leq p(0) .
$$

We show that $n_{1}(\lambda) \leq p(\lambda)$ for all $\lambda \in \mathbb{R}$. To do so, let (as in Section 4) $\lambda_{\mu}$ denote the finite eigenvalues of $(\mathrm{E})$ with corresponding orthonormal eigenvectors $z^{(\mu)}$ for $1 \leq \mu \leq r$ such that $\lambda_{1} \leq \ldots \leq \lambda_{r}$. Now, given $\lambda \in \mathbb{R}$, we have that

$$
\lambda_{m} \leq \lambda<\lambda_{m+1} \quad \text { for some } m \in\{0, \ldots, r\},
$$

where we put $\lambda_{0}=-\infty$ and $\lambda_{r+1}=\infty$. By (2.1), this means that $m=n_{1}(\lambda)$. First suppose that $\lambda$ is not a finite eigenvalue of (E) so that $\lambda_{m}<\lambda<\lambda_{m+1}$. Put $\tilde{z}=\sum_{\mu=1}^{m} \beta_{\mu} z^{(\mu)}$, where the constants $\beta_{1}, \beta_{2}, \ldots, \beta_{m}$ are chosen in such a way that $\tilde{z}=(\tilde{x}, \tilde{u})$ satisfies $\tilde{p}$ linear homogeneous conditions

$$
\left\{\begin{aligned}
\underset{\tilde{s}_{k}}{\sim} \tilde{x}_{k+1}=0, \quad k \in\{0, \ldots, N-1\}, & \\
\tilde{s}_{k} \perp\left\{\alpha \in \mathbb{R}^{n}: \quad\right. & \alpha \text { is an eigenvector corresponding } \\
& \text { to a negative eigenvalue of } \underset{\sim}{P}\}, \quad k \in\{0, \ldots, N\},
\end{aligned}\right.
$$

where

$$
\tilde{s}_{k}=\tilde{u}_{k}-Q_{k} \tilde{x}_{k}=\sum_{\mu=1}^{m} \beta_{\mu}\left(\tilde{u}_{k}^{(\mu)}-Q_{k} \tilde{x}_{k}^{(\mu)}\right)
$$

with the matrix $Q$ satisfying $Q \underset{\sim}{X}=\underset{\sim}{U} \underset{\sim}{X} \underset{\sim}{X}$ and the matrices $\underset{\sim}{M}, \underset{\sim}{P}$ given by (1.1) with $(X, U)=(\underset{\sim}{X}, \underset{\sim}{U})$, and where $\tilde{p}$ equals to the number of focal points of $(\underset{\sim}{X}, \underset{\sim}{U})$ in the open interval $(0, N+1)$ so that $\tilde{p} \leq p$. The sequence $\tilde{z}$ is admissible for $\mathcal{F}$, and by the second condition in (1.4) there exists $\underset{\sim}{u}$ such that $(\tilde{x}, \underset{\sim}{u})$ is admissible for $\underset{\sim}{\mathcal{F}}$. Since the value of the quadratic functional does not depend on the second component of an admissible sequence $z=(x, u)$ (see Lemma 2.1), we also write $\tilde{z}=(\tilde{x}, u)$. Then by the extended Picone identity, Theorem 4.2 from Section 4 , we have

$$
\mathcal{F}_{\sim}(\tilde{z}):=\underset{\sim}{\mathcal{F}}(\tilde{z})-\lambda\langle\tilde{z}, \tilde{z}\rangle=\sum_{k=0}^{N} \tilde{s}_{k}^{T}{\underset{\sim}{k}}_{k} \tilde{s}_{k} \geq 0
$$


Note that the first condition in (2.3) implies that $\tilde{x}_{k} \in \operatorname{Im} \underset{\sim}{X} X_{k}$ for all $k \in\{0, \ldots, N+$ 1 ) by Proposition 4.4 from Section 4 , and hence Theorem 4.2 can be applied. At the same time, by a direct computation using orthonormality of $z^{(1)}, z^{(2)}, \ldots, z^{(m)}$ (see also Section 4), we have

$$
\mathcal{F}_{\lambda}(\tilde{z}):=\mathcal{F}(\tilde{z})-\lambda\langle\tilde{z}, \tilde{z}\rangle=\sum_{\mu=1}^{m}\left(\lambda_{\mu}-\lambda\right) \beta_{\mu}^{2} .
$$

Now by Lemma 2.1 and the first condition in (1.4), we have

$$
\sum_{\mu=1}^{m}\left(\lambda_{\mu}-\lambda\right) \beta_{\mu}^{2} \geq \sum_{k=0}^{N} \tilde{s}_{k}^{T} P_{k} \tilde{s}_{k} \geq 0
$$

since $\lambda>\lambda_{\mu}$ for all $1 \leq \mu \leq m$; this is possible only if $\beta_{\mu}=0$ for all $1 \leq \mu \leq m$. This means that the system of $\tilde{p}$ linear homogeneous conditions (2.3) has only the trivial solution, and hence the number of conditions $\tilde{p}$ is greater than or equal to the number of parameters $\beta_{\mu}$ which is $m$. This proves the statement when $\lambda$ is not a finite eigenvalue of $(\mathrm{E})$. Since the functions $n_{1}(\lambda)$ and $p(\lambda)$ are continuous from the right by (4.8), by letting $\lambda \rightarrow \lambda_{m}^{+}$we obtain the statement also in the case when $\lambda=\lambda_{m}$ is a finite eigenvalue of (E).

\section{Sturmian SEPARATion RESUlts}

Combining Theorems 1.2 and 1.3 , we obtain the following statement. This result extends [11, Theorem 1], where the statement is proved in the special case $m=0$ (see also [7, Theorem 1]).

Theorem 3.1. If the principal solution of $(\underline{S})$ has $m$ focal points in $(0, N+1]$, then any conjoined basis of (S) has at least $m$ and at most $m+n$ focal points in $(0, N+1]$.

Proof. We apply Theorems 1.2 and 1.3 with $\underset{\sim}{\mathcal{S}}=\mathcal{S}$ and note that the assumptions in both theorems are satisfied since $\underset{\sim}{\mathcal{G}}=\mathcal{G}$ and $\operatorname{Im}\left(\begin{array}{ll}0 & \mathcal{B}\end{array}\right) \subset \operatorname{Im} \mathcal{B}$ imply that (1.4) holds.

Now we prove Theorem 1.1 as stated in the Introduction of this paper.

Proof of Theorem 1.1. Suppose that $Z$ and $\tilde{Z}$ are two conjoined bases of (SI) with $p$ and $\tilde{p}$ focal points in $(0, N+1]$, respectively. Theorem 3.1 yields that

$$
m \leq p \leq m+n \quad \text { and } \quad m \leq \tilde{p} \leq m+n,
$$

where $m$ is the number of focal points of the principal solution in $(0, N+1]$. This yields the assertion.

Next we show that using the construction introduced in [9, page 1256] we can derive a more precise estimate for the difference of the numbers of focal points of the principal solution of (SS) and of any other conjoined basis of $(\underline{S})$. This statement can be regarded as a discrete version of [22, Problems VII.7, problem 2, page 367].

Theorem 3.2. Let $(\tilde{X}, \tilde{U})$ be the principal solution of $(\underline{S})$ and let $(X, U)$ be any conjoined basis of this system. Let $m$ and $p$ denote the number of focal points of $(\tilde{X}, \tilde{U})$ and of $(X, U)$ in $(0, N+1]$, respectively. Then

$$
m \leq p \leq m+\operatorname{rank} X_{0} .
$$


Proof. We extend the eigenvalue problem $(\mathrm{E})$ to a problem of the same kind on the interval $[-1, N+1]$. Define

$$
W_{-1}:=I \quad \text { and } \quad \mathcal{S}_{-1}:=\left(\begin{array}{ll}
\mathcal{A}_{-1} & \mathcal{B}_{-1} \\
\mathcal{C}_{-1} & \mathcal{D}_{-1}
\end{array}\right):=\left(\begin{array}{cc}
U_{0} K & X_{0} \\
-X_{0} K & U_{0}
\end{array}\right),
$$

where $K:=\left(X_{0}^{T} X_{0}+U_{0}^{T} U_{0}\right)^{-1}$. Then by a direct computation we see that the matrix $S_{-1}$ is symplectic and hence

$\left\{\begin{array}{l}x_{k+1}=\mathcal{A}_{k} x_{k}+\mathcal{B}_{k} u_{k}, \quad u_{k+1}=\mathcal{C}_{k} x_{k}+\mathcal{D}_{k} u_{k}-\lambda x_{k+1}, \quad k \in\{-1,0,1, \ldots, N\}, \\ x_{-1}=x_{N+1}=0\end{array}\right.$

is an eigenvalue problem for a symplectic system. Now we also extend $(\tilde{X}, \tilde{U})$ and $(X, U)$ to $[-1, N+1]$ by

$$
\left(\begin{array}{l}
\tilde{X}_{-1} \\
\tilde{U}_{-1}
\end{array}\right):=S_{-1}^{-1}\left(\begin{array}{l}
\tilde{X}_{0} \\
\tilde{U}_{0}
\end{array}\right)=\left(\begin{array}{cc}
U_{0}^{T} & -X_{0}^{T} \\
K X_{0}^{T} & K U_{0}^{T}
\end{array}\right)\left(\begin{array}{l}
0 \\
I
\end{array}\right)=\left(\begin{array}{c}
-X_{0}^{T} \\
K U_{0}^{T}
\end{array}\right)
$$

and

$$
\left(\begin{array}{l}
X_{-1} \\
U_{-1}
\end{array}\right):=S_{-1}^{-1}\left(\begin{array}{l}
X_{0} \\
U_{0}
\end{array}\right)=\left(\begin{array}{cc}
U_{0}^{T} & -X_{0}^{T} \\
K X_{0}^{T} & K U_{0}^{T}
\end{array}\right)\left(\begin{array}{l}
X_{0} \\
U_{0}
\end{array}\right)=\left(\begin{array}{l}
0 \\
I
\end{array}\right)
$$

Hence

$$
\tilde{M}_{-1}=\left(I-\tilde{X}_{0} \tilde{X}_{0}^{\dagger}\right) \mathcal{B}_{-1}=X_{0}, \tilde{T}_{-1}=I-X_{0}^{\dagger} X_{0}, \tilde{P}_{-1}=\tilde{T}_{-1}^{T} \tilde{X}_{-1} \tilde{X}_{0}^{\dagger} \mathcal{B}_{-1} \tilde{T}_{-1}=0,
$$

which means that the extended $(\tilde{X}, \tilde{U})$ has $\operatorname{rank} X_{0}$ focal points in $(-1,0]$ and, since $X_{-1}=0$, the extended conjoined basis $(X, U)$ has no additional focal point in $(-1,0]$. Denote by $\hat{m}$ and $\hat{p}$ the number of focal points of the extended $(\tilde{X}, \tilde{U})$ and $(X, U)$ in $(-1, N+1]$, respectively. Then $\hat{m}=m+\operatorname{rank} X_{0}, \hat{p}=p$, and by Theorem 1.3 and $\mathcal{\sim}=\mathcal{S}$ applied once to $(\underline{S})$ on $[0, N+1]$ and once on $[-1, N+1]$ (with $S_{-1}$ defined by (3.1)), we obtain

$$
m \leq p=\hat{p} \leq \hat{m}=m+\operatorname{rank} X_{0},
$$

which completes the proof.

\section{Spectral theory}

We consider the (slightly more general than in Section 1) symplectic eigenvalue problem associated with (프),

$$
\left\{\begin{array}{l}
x_{k+1}=\mathcal{A}_{k} x_{k}+\mathcal{B}_{k} u_{k}, \quad u_{k+1}=\mathcal{C}_{k} x_{k}+\mathcal{D}_{k} u_{k}-\lambda \mathcal{W}_{k} x_{k+1}, \quad k \in\{0, \ldots, N\}, \\
x_{0}=x_{N+1}=0,
\end{array}\right.
$$

where we assume that

$$
\mathcal{W}_{k} \geq 0 .
$$

The following definition is as in [12, Definitions 2 and 3, Proposition 2 (v)]. 
Definition 4.1. A number $\lambda \in \mathbb{R}$ is called a finite eigenvalue of (4.1) provided it possesses a corresponding finite eigenvector $z=(x, u)$, i.e., $z$ solves (4.1) such that $\left\{\mathcal{W}_{k} x_{k+1}\right\}_{k=0}^{N-1} \not \equiv 0$, and then

$$
\operatorname{dim}\left\{\left\{\mathcal{W}_{k} x_{k+1}\right\}_{k=0}^{N-1}: z=(x, u) \text { solves (4.1) }\right\}
$$

is called its multiplicity.

By [12, Proposition 2], finite eigenvectors corresponding to different finite eigenvalues are orthogonal with respect to the bilinear form

$$
\langle z, \tilde{z}\rangle_{\mathcal{W}}:=\langle z, \tilde{z}\rangle:=\sum_{k=0}^{N} x_{k+1}^{T} \mathcal{W}_{k} \tilde{x}_{k+1} \quad \text { for } \quad z=(x, u) \text { and } \tilde{z}=(\tilde{x}, \tilde{u}) .
$$

Moreover, we consider the discrete bilinear form $\mathcal{F}_{\lambda}$ associated with (4.1),

$$
\mathcal{F}_{\lambda}(z, \tilde{z})=\sum_{k=0}^{N}\left\{x_{k}^{T} \mathcal{A}_{k}^{T} \mathcal{C}_{k} \tilde{x}_{k}+x_{k}^{T} \mathcal{C}_{k}^{T} \mathcal{B}_{k} \tilde{u}_{k}+u_{k}^{T} \mathcal{B}_{k}^{T} \mathcal{C}_{k} \tilde{x}_{k}+u_{k}^{T} \mathcal{B}_{k}^{T} \mathcal{D}_{k} \tilde{u}_{k}\right\}-\lambda\langle z, \tilde{z}\rangle_{\mathcal{W}}
$$

for admissible $z=(x, u)$ and $\tilde{z}=(\tilde{x}, \tilde{u})$, and the quadratic functional $\mathcal{F}_{\lambda}(z)=$ $\mathcal{F}_{\lambda}(z, z)$, so that $\mathcal{F}_{0}(z)=\mathcal{F}(z)$ with the notation of Section 2 above.

4.1. Picone's identity. We extend the generalized Picone identity from [10, Proposition 2.1] as follows. For the continuous version of this identity, see [17, Theorem 2.2.3].

Theorem 4.2 (Extended Picone identity). Suppose that $Z=(X, U)$ is a conjoined basis of the symplectic system of (4.1) for a fixed $\lambda \in \mathbb{R}$, let $Q$ be symmetric with $Q X=U X^{\dagger} X$, and define $M, T$, and $P$ by (1.1). Let $\lambda_{1}, \ldots, \lambda_{m}$ be finite eigenvalues with corresponding orthonormal finite eigenfunctions $z^{(\mu)}=\left(x^{(\mu)}, u^{(\mu)}\right), 1 \leq$ $\mu \leq m$, with respect to $\langle\cdot, \cdot\rangle_{\mathcal{W}}$, and let $\beta_{1}, \ldots, \beta_{m} \in \mathbb{R}$ and put $\hat{z}:=\sum_{\mu=1}^{m} \beta_{\mu} z^{(\mu)}$. Finally, suppose that $z=(x, u)$ is admissible, put $\tilde{z}:=z+\hat{z}, \tilde{s}:=\tilde{u}-Q \tilde{x}$, and assume that

$$
z \perp z^{(\mu)}, \quad \text { i.e., } \quad\left\langle z, z^{(\mu)}\right\rangle_{\mathcal{W}}=0 \text { for } 1 \leq \mu \leq m
$$

and that

$$
\tilde{x}_{k} \in \operatorname{Im} X_{k} \quad \text { for all } \quad k \in\{0, \ldots, N+1\} .
$$

Then we have that

$\mathcal{F}_{\lambda}(z)-\left.x_{k}^{T} u_{k}\right|_{k=0} ^{N+1}=\sum_{k=0}^{N} \tilde{s}_{k}^{T} P_{k} \tilde{s}_{k}+\sum_{\mu=1}^{m}\left(\lambda-\lambda_{\mu}\right)\left|\beta_{\mu}\right|^{2}+\left.\tilde{x}_{k}^{T} Q_{k} \tilde{x}_{k}\right|_{k=0} ^{N+1}-\left.\tilde{x}_{k}^{T} \tilde{u}_{k}\right|_{k=0} ^{N+1}$.

Proof. First, we obtain from [10, Proposition 2.1 (iv)] (observe that assumption (1.7) is not needed there) that

$$
\begin{aligned}
\mathcal{F}_{\lambda}(\tilde{z}) & =\sum_{k=0}^{N}\left\{\tilde{x}_{k+1}^{T} \tilde{Q}_{k} \tilde{x}_{k+1}-\tilde{x}_{k}^{T} \tilde{Q}_{k} \tilde{x}_{k}+\tilde{s}_{k}^{T} P_{k} \tilde{s}_{k}\right\} \\
& =\left.\tilde{x}_{k}^{T} Q_{k} \tilde{x}_{k}\right|_{k=0} ^{N+1}+\sum_{k=0}^{N} \tilde{s}_{k}^{T} P_{k} \tilde{s}_{k}
\end{aligned}
$$


because $\tilde{z}$ is admissible and (4.4) holds. Next, from [8, page 711] or [11, Lemma 1], from the recursion of (4.1) for $\lambda=\lambda_{\mu}, 1 \leq \mu \leq m$, and from orthonormality we conclude that

$$
\begin{aligned}
\mathcal{F}_{0}(\hat{z}) & =\left.\hat{x}_{k}^{T} \hat{u}_{k}\right|_{k=0} ^{N+1}+\sum_{k=0}^{N} \hat{x}_{k+1}^{T}\left\{\mathcal{C}_{k} \hat{x}_{k}+\mathcal{D}_{k} \hat{u}_{k}-\hat{u}_{k+1}\right\} \\
& =\left.\hat{x}_{k}^{T} \hat{u}_{k}\right|_{k=0} ^{N+1}+\sum_{\mu=1}^{m} \sum_{k=0}^{N} \hat{x}_{k+1}^{T} \beta_{\mu} \lambda_{\mu} x_{k+1}^{(\mu)} \\
& =\left.\hat{x}_{k}^{T} \hat{u}_{k}\right|_{k=0} ^{N+1}+\sum_{\mu=1}^{m} \lambda_{\mu}\left|\beta_{\mu}\right|^{2}
\end{aligned}
$$

and using (4.3),

$$
\mathcal{F}_{0}(z, \hat{z})=\left.x_{k}^{T} \hat{u}_{k}\right|_{k=0} ^{N+1}+\sum_{\mu=1}^{m} \sum_{k=0}^{N} x_{k+1}^{T} \beta_{\mu} \lambda_{\mu} x_{k+1}^{(\mu)}=\left.x_{k}^{T} \hat{u}_{k}\right|_{k=0} ^{N+1}
$$

and

Altogether, we can conclude that

$$
\mathcal{F}_{0}(\hat{z}, z)=\left.\hat{x}_{k}^{T} u_{k}\right|_{k=0} ^{N+1} .
$$

$$
\begin{aligned}
& \mathcal{F}_{\lambda}(z)-\left.x_{k}^{T} u_{k}\right|_{k=0} ^{N+1}=\mathcal{F}_{0}(z)-\lambda\langle z, z\rangle_{\mathcal{W}}-\left.x_{k}^{T} u_{k}\right|_{k=0} ^{N+1} \\
& \quad=\mathcal{F}_{0}(\tilde{z})-\mathcal{F}_{0}(\hat{z})-\mathcal{F}_{0}(z, \hat{z})-\mathcal{F}_{0}(\hat{z}, z)-\lambda\langle z, z\rangle_{\mathcal{W}}-\left.x_{k}^{T} u_{k}\right|_{k=0} ^{N+1} \\
& =\mathcal{F}_{\lambda}(\tilde{z})+\lambda\langle z+\hat{z}, z+\hat{z}\rangle_{\mathcal{W}}-\mathcal{F}_{0}(\hat{z})-\left.\left(x_{k}^{T} \hat{u}_{k}+\hat{x}_{k}^{T} u_{k}+x_{k}^{T} u_{k}\right)\right|_{k=0} ^{N+1} \\
& =\sum_{k=0}^{N} \tilde{s}_{k}^{T} P_{k} \tilde{s}_{k}+\left.\tilde{x}_{k}^{T} Q_{k} \tilde{x}_{k}\right|_{k=0} ^{N+1}-\left.\tilde{x}_{k}^{T} \tilde{u}_{k}\right|_{k=0} ^{N+1}-\sum_{\mu=1}^{m} \lambda_{\mu}\left|\beta_{\mu}\right|^{2}+\lambda\langle\hat{z}, \hat{z}\rangle_{\mathcal{W}}
\end{aligned}
$$

using the fact that $z \perp \hat{z}$ by (4.3), and orthonormality yields our assertion (4.5).

Note first that the existence of a matrix $Q$ with the requirements as in Theorem 4.2 is established in 8. Next we analyze the crucial assumption (4.4) using notation (1.1).

Lemma 4.3. Suppose that $Z=(X, U)$ is a conjoined basis of the symplectic system $(\underline{S})$ and let $0 \leq k \leq N$. Then we have

(i) $x_{k+1} \in \operatorname{Im} X_{k+1}$ implies that $M_{k}^{T} x_{k+1}=0$;

(ii) $x_{k+1}=\mathcal{A}_{k} x_{k}+\mathcal{B}_{k} u_{k}, M_{k}^{T} x_{k+1}=0$, and $x_{k} \in \operatorname{Im} X_{k}$ imply that $x_{k+1} \in$ $\operatorname{Im} X_{k+1}$.

Proof. First, $x_{k+1}=X_{k+1} c \in \operatorname{Im} X_{k+1}$ implies by (1.1) that

$$
M_{k}^{T} x_{k+1}=\mathcal{B}_{k}^{T}\left(I-X_{k+1} X_{k+1}^{\dagger}\right) X_{k+1} c=0 .
$$

Hence, (i) is true. Next, $x_{k+1}=\mathcal{A}_{k} x_{k}+\mathcal{B}_{k} u_{k}, x_{k}=X_{k} c \in \operatorname{Im} X_{k}$, and $M_{k}^{T} x_{k+1}=0$ imply that

$$
\begin{aligned}
0 & =\mathcal{B}_{k}^{T}\left(I-X_{k+1} X_{k+1}^{\dagger}\right)\left(\mathcal{A}_{k} X_{k} c+\mathcal{B}_{k} u_{k}\right) \\
& =\mathcal{B}_{k}^{T}\left(I-X_{k+1} X_{k+1}^{\dagger}\right)\left(X_{k+1} c+\mathcal{B}_{k}\left(u_{k}-U_{k} c\right)\right) \\
& =\mathcal{B}_{k}^{T}\left(I-X_{k+1} X_{k+1}^{\dagger}\right) \mathcal{B}_{k}\left(u_{k}-U_{k} c\right) \\
& =\mathcal{B}_{k}^{T}\left(I-X_{k+1} X_{k+1}^{\dagger}\right)^{T}\left(I-X_{k+1} X_{k+1}^{\dagger}\right) \mathcal{B}_{k}\left(u_{k}-U_{k} c\right)
\end{aligned}
$$


so that

$$
0=\left(I-X_{k+1} X_{k+1}^{\dagger}\right) \mathcal{B}_{k}\left(u_{k}-U_{k} c\right)
$$

and therefore

$$
x_{k+1}=X_{k+1} c+\mathcal{B}_{k}\left(u_{k}-U_{k} c\right)=X_{k+1} c+X_{k+1} X_{k+1}^{\dagger} \mathcal{B}_{k}\left(u_{k}-U_{k} c\right) \in \operatorname{Im} X_{k+1}
$$

holds. Hence, (ii) is true.

Proposition 4.4. Suppose that $Z=(X, U)$ is a conjoined basis of the symplectic system (S) and let $z=(x, u)$ be admissible with $x_{0}=x_{N+1}=0$. Then (4.4) holds for $\tilde{x}=x$ if and only if

$$
M_{k}^{T} x_{k+1}=0 \quad \text { for all } \quad 0 \leq k \leq N-1 .
$$

Proof. First, (4.4) implies (4.6) by Lemma 4.3 (i). Next, suppose that $z=(x, u)$ is admissible, $x_{0}=x_{N+1}=0$, and (4.6) holds. Then $x_{0}=0 \in \operatorname{Im} X_{0}$, and inductively $x_{k+1} \in \operatorname{Im} X_{k+1}$ for all $0 \leq k \leq N-1$. Moreover, $x_{N+1}=0 \in \operatorname{Im} X_{N+1}$, so that (4.4) holds for $\tilde{x}=x$.

4.2. Rayleigh's principle. In this subsection we put $\langle\cdot, \cdot\rangle=\langle\cdot, \cdot\rangle_{\mathcal{W}}$ and assume throughout that

$$
\mathcal{W}_{k}=I \quad \text { for all } \quad 0 \leq k \leq N
$$

(as in Sections 2 and 3) and that $Z(\lambda)=(X(\lambda), U(\lambda))$ is the principal solution of the symplectic system in (E), i.e., that

$$
X_{0}=X_{0}(\lambda) \equiv 0 \quad \text { and } \quad U_{0}=U_{0}(\lambda) \equiv I .
$$

We need the following lemma.

Lemma 4.5. There exists $\lambda_{0} \in \mathbb{R}$ such that $\lambda \leq \lambda_{0}$ implies $\mathcal{F}_{\lambda}(z)>0$ for all admissible $z=(x, u)$ with $x_{0}=x_{N+1}=0$ and $x=\left\{x_{k}\right\}_{k=0}^{N} \not \equiv 0$.

Proof. Let $z$ be admissible with $x_{0}=x_{N+1}=0$. Then, by (4.2) and (4.7),

$$
\begin{aligned}
\mathcal{F}_{\lambda}(z)= & \sum_{k=0}^{N}\left\{x_{k}^{T} \mathcal{A}_{k}^{T} \mathcal{C}_{k} x_{k}+2 x_{k}^{T} \mathcal{C}_{k}^{T}\left(x_{k+1}-\mathcal{A}_{k} x_{k}\right)\right. \\
& \left.\quad+\left(x_{k+1}-\mathcal{A}_{k} x_{k}\right)^{T} \mathcal{B}_{k} \mathcal{B}_{k}^{\dagger} \mathcal{D}_{k} \mathcal{B}_{k}^{\dagger}\left(x_{k+1}-\mathcal{A}_{k} x_{k}\right)-\lambda x_{k+1}^{T} x_{k+1}\right\} \\
\geq & \sum_{k=1}^{N}\left\{x_{k}^{T} \mathcal{F}_{k} x_{k}-\lambda\left|x_{k}\right|^{2}\right\}
\end{aligned}
$$

for certain matrices $\mathcal{F}_{k}$. This yields the assertion if $\lambda_{0}$ is sufficiently small.

Now, using Lemma 4.5, we obtain from [12, Theorem 2]

$$
n_{1}(\lambda)=n_{2}(\lambda), n_{1}(\lambda+)=n_{1}(\lambda), n_{2}(\lambda+)=n_{2}(\lambda) \quad \text { for all } \quad \lambda \in \mathbb{R},
$$

where, including multiplicities,

$n_{1}(\lambda)$ denotes the number of focal points of $Z=Z(\lambda)$ in the interval $(0, N+1]$;

$n_{2}(\lambda)$ denotes the number of finite eigenvalues of $(\mathbb{E})$ which are less than or equal to $\lambda$. 
We let (see [12, Proposition 2])

$\lambda_{1} \leq \ldots \leq \lambda_{r} \quad$ denote the finite eigenvalues of $(\mathbb{E})$,

including multiplicities, with corresponding orthonormal finite eigenfunctions $z^{(\mu)}$, $1 \leq \mu \leq r$, and we put $\lambda_{0}:=-\infty$ and $\lambda_{r+1}:=\infty$. Then, by [12, Proposition 2], $r \leq n N<\infty$.

Theorem 4.6 (Rayleigh principle). With the above notation and assumptions we have for $0 \leq m \leq r$ that

$$
\begin{aligned}
\lambda_{m+1}=\min \left\{\frac{\mathcal{F}_{0}(z)}{\langle z, z\rangle}: \quad\right. & z=(x, u) \text { is admissible with } x_{0}=x_{N+1}=0, \\
& \left.z \perp z^{(\mu)} \text { for all } 1 \leq \mu \leq m, \text { and } x=\left\{x_{k}\right\}_{k=1}^{N} \not \equiv 0\right\} .
\end{aligned}
$$

Note that we include the cases $m=0$, where the orthogonality condition on $z$ becomes empty, and $m=r$, where $\lambda_{m+1}=\infty$.

Proof. Let $0 \leq m \leq r$ and $\lambda \in\left(\lambda_{m}, \lambda_{m+1}\right)$. Then, by (4.8),

$$
m=n_{1}(\lambda)=n_{2}(\lambda)
$$

so the principal solution possesses exactly $m$ focal points in the open interval $(0, N+$ 1 ), because $N+1$ is not a focal point. The fact that $N+1$ is not a focal point follows from Lemma 4.5, which implies that $\operatorname{rank} M_{k}(\tilde{\lambda})=0$ for all $\tilde{\lambda} \leq \lambda_{0}$ so that by [9, Remark 3 (ii)]

$$
\operatorname{rank} M_{k}(\lambda+)=\operatorname{rank} M_{k}(\lambda)=0 \quad \text { for all } \quad 0 \leq k \leq N, \lambda \in\left(\lambda_{m}, \lambda_{m+1}\right),
$$

in particular rank $M_{N}(\lambda)=0$.

First, we apply the extended Picone identity, Theorem 4.2, to $z=0$ so that

$$
\tilde{z}=\hat{z}=\sum_{\mu=1}^{m} \beta_{\mu} z^{(\mu)} \quad \text { with } \quad \tilde{x}_{0}=\tilde{x}_{N+1}=x_{0}=x_{N+1}=0 .
$$

We use an argument similar to that applied in the proof of Theorem 1.3. Suppose that $\beta_{1}, \ldots, \beta_{m}$ satisfy the $m$ linear and homogeneous equations

$$
\left\{\begin{aligned}
& M_{k}^{T} \tilde{x}_{k+1}=0, \quad k \in\{0, \ldots, N-1\}, \\
& \tilde{s}_{k} \perp\left\{\alpha \in \mathbb{R}^{n}: \quad \alpha\right. \text { is an eigenvector corresponding } \\
&\text { to a negative eigenvalue of } \left.P_{k}\right\}, \quad k \in\{0, \ldots, N\},
\end{aligned}\right.
$$

where

$$
\tilde{s}_{k}=\tilde{u}_{k}-Q_{k} \tilde{x}_{k}
$$

Note that the number of these equations is just the number of focal points in $(0, N+1)$ by definition. Then, by Proposition 4.4, the assumption (4.4) holds, and we obtain from Theorem 4.2 that

$$
0=\mathcal{F}_{\lambda}(z)-\left.x_{k}^{T} u_{k}\right|_{k=0} ^{N+1}=\sum_{k=0}^{N} \tilde{s}_{k}^{T} P_{k} \tilde{s}_{k}+\sum_{\mu=1}^{m}\left(\lambda-\lambda_{\mu}\right)\left|\beta_{\mu}\right|^{2},
$$

where $\tilde{s}_{k}^{T} P_{k} \tilde{s}_{k} \geq 0$ for $0 \leq k \leq N$ by (4.9), and $\lambda-\lambda_{\mu} \geq \lambda-\lambda_{m}>0$ for $1 \leq \mu \leq m$. Hence $\beta_{1}=\cdots=\beta_{m}=0$ so that (4.9) possesses only the trivial solution. Thus we have shown that

$$
\text { the coefficient matrix corresponding to (4.9) is nonsingular. }
$$


Now suppose that $z$ is admissible with $x_{0}=x_{N+1}=0$ and $z \perp z^{(\mu)}$ for $1 \leq$ $\mu \leq m$. Then we apply Theorem 4.2 to $\tilde{z}=z+\hat{z}=z+\sum_{\mu=1}^{m} \beta_{\mu} z^{(\mu)}$, where we choose $\beta_{1}, \ldots, \beta_{m}$ such that (4.9) holds. This is possible because the $m$ linear and inhomogeneous equations possess a unique solution by (4.10). Theorem 4.2 implies that

$$
\begin{aligned}
\mathcal{F}_{\lambda}(z) & =\mathcal{F}_{\lambda}(z)-\left.x_{k}^{T} u_{k}\right|_{k=0} ^{N+1} \\
& =\sum_{k=0}^{N} \tilde{s}_{k}^{T} P_{k} \tilde{s}_{k}+\sum_{\mu=1}^{m}\left(\lambda-\lambda_{\mu}\right)\left|\beta_{\mu}\right|^{2} \\
& \geq \sum_{\mu=1}^{m}\left(\lambda-\lambda_{\mu}\right)\left|\beta_{\mu}\right|^{2} \geq 0
\end{aligned}
$$

so that

$$
\mathcal{F}_{0}(z) \geq \lambda\langle z, z\rangle \quad \text { for all } \quad \lambda \in\left(\lambda_{m}, \lambda_{m+1}\right) .
$$

Hence $\mathcal{F}_{0}(z) \geq \lambda_{m+1}\langle z, z\rangle$, and $\mathcal{F}_{0}(z)=\lambda_{m+1}\langle z, z\rangle$ for $z=z^{(m+1)}$. For multiple finite eigenvalues use the fact that $\mathcal{F}_{\lambda}(z)=\mathcal{F}_{\lambda}(z+\hat{z})$ if $z$ is admissible with $x_{0}=$ $x_{N+1}=0$ and if $\hat{z}$ is a finite eigenvector corresponding to $\lambda$. Hence the assertion follows.

Rayleigh's principle yields the following result.

Theorem 4.7 (Expansion theorem). Suppose that $z=(x, u)$ is admissible with $x_{0}=x_{N+1}=0$. Then

$$
x=\sum_{\mu=1}^{r} c_{\mu} x^{(\mu)}, \quad \text { where } \quad c_{\mu}=\left\langle z^{(\mu)}, z\right\rangle .
$$

Proof. First, suppose that $z$ is admissible with $x_{0}=x_{N+1}=0$ and $z \perp z^{(\mu)}$ for all $1 \leq \mu \leq r$. Then, by Theorem 4.6 with $m=r, \mathcal{F}_{0}(z) \geq \lambda\langle z, z\rangle$ for all $\lambda \in \mathbb{R}$. Hence

$$
\langle z, z\rangle=\sum_{k=0}^{N} x_{k+1}^{T} x_{k+1}=0 \quad \text { so that } \quad x=0 .
$$

Now if $z$ is admissible with $x_{0}=x_{N+1}=0$, then $z-\sum_{\mu=1}^{r}\left\langle z^{(\mu)}, z\right\rangle z^{(\mu)} \perp z^{(m)}$ for all $1 \leq m \leq r$, and (4.11) follows from what we have shown before.

In our final result we prove that "extremal vectors of the functional $\mathcal{F}_{0}$ satisfy necessarily the corresponding Euler equations". The meaning of this becomes clear from the formulation of the following theorem.

Theorem 4.8. Let be given any eigenvalue problem (E) with (4.7) and with corresponding functional $\mathcal{F}_{\lambda}$, and let $m \in\{0, \ldots, r\}$. Suppose that

$$
\begin{aligned}
\mathcal{F}_{0}(\hat{z})=\lambda_{m+1}\langle\hat{z}, \hat{z}\rangle \quad & \text { for some admissible } \hat{z} \text { with } \hat{x}_{0}=\hat{x}_{N+1}=0 \\
& \text { and } \hat{z} \perp z^{(\mu)} \text { for all } 1 \leq \mu \leq m .
\end{aligned}
$$

Then $\hat{z}$ satisfies the Euler equation, i.e.,

$$
\left\{\begin{array}{l}
\tilde{u}_{k+1}=\mathcal{C}_{k} \hat{x}_{k}+\mathcal{D}_{k} \tilde{u}_{k}-\lambda_{m+1} \hat{x}_{k+1} \text { for suitable vectors } \tilde{u}_{k} \text { with } \\
\mathcal{B}_{k} \tilde{u}_{k}=\mathcal{B}_{k} \hat{u}_{k} \text { for } 0 \leq k \leq N .
\end{array}\right.
$$


Proof. Let $\hat{z}$ be admissible with $\hat{x}_{0}=\hat{x}_{N+1}=0, \hat{z} \perp z^{(\mu)}$ for all $1 \leq \mu \leq m$, and $\mathcal{F}_{0}(\hat{z})=\lambda_{m+1}\langle\hat{z}, \hat{z}\rangle$. Then, by the expansion theorem, Theorem 4.7 $\hat{x}=$ $\sum_{\mu=1}^{r} c_{\mu} x^{(\mu)}, c_{\mu}=\left\langle z^{(\mu)}, \hat{z}\right\rangle$, where $c_{\mu}=0$ for $1 \leq \mu \leq m$, by using the notation above. It follows that (use e.g., Theorem 4.2)

$$
\lambda_{m+1}\langle\hat{z}, \hat{z}\rangle=\mathcal{F}_{0}(\hat{z})=\sum_{\mu=1}^{r} \lambda_{\mu}\left|c_{\mu}\right|^{2} \geq \lambda_{m+1} \sum_{\mu=m+1}^{r}\left|c_{\mu}\right|^{2}=\lambda_{m+1}\langle\hat{z}, \hat{z}\rangle
$$

so that $c_{\mu}=0$ for all $\mu \geq \ell+1$, where $\lambda_{m+1}=\ldots=\lambda_{\ell}<\lambda_{\ell+1}$. Hence $\tilde{z}:=$ $\sum_{\mu=m+1}^{\ell} c_{\mu} z^{(\mu)}$ is an eigenfunction corresponding to $\lambda_{m+1}$ with $\tilde{x}=\hat{x}$. Then (4.13) holds because

$$
\tilde{u}_{k+1}=\mathcal{C}_{k} \tilde{x}_{k}+\mathcal{D}_{k} \tilde{u}_{k}-\lambda_{m+1} \tilde{x}_{k+1}=\mathcal{C}_{k} \hat{x}_{k}+\mathcal{D}_{k} \tilde{u}_{k}-\lambda_{m+1} \hat{x}_{k+1}
$$

and

$$
\mathcal{B}_{k}\left(\tilde{u}_{k}-\hat{u}_{k}\right)=\left(\tilde{x}_{k+1}-\hat{x}_{k+1}\right)-\mathcal{A}_{k}\left(\tilde{x}_{k}-\hat{x}_{k}\right)=0 .
$$

The proof is complete.

\section{REFERENCES}

[1] R. P. Agarwal, M. Bohner, S. R. Grace, and D. O'Regan. Discrete Oscillation Theory. Hindawi Publishing Corporation, 2005.

[2] C. D. Ahlbrandt and A. C. Peterson. Discrete Hamiltonian Systems: Difference Equations, Continued Fractions, and Riccati Equations, volume 16 of Kluwer Texts in the Mathematical Sciences. Kluwer Academic Publishers, Boston, 1996. MR1423802 (98m:39043)

[3] A. Ben-Israel and T. N. E. Greville. Generalized Inverses: Theory and Applications. John Wiley \& Sons, Inc., New York, 1974. MR0396607 (53:469)

[4] M. Bohner. Controllability and disconjugacy for linear Hamiltonian difference systems. In S. Elaydi, J. Graef, G. Ladas, and A. Peterson, editors, Conference Proceedings of the First International Conference on Difference Equations, pages 65-77, San Antonio, 1994. Gordon and Breach. MR1678645

[5] M. Bohner. Zur positivität diskreter quadratischer Funktionale. Ph.D. thesis, Universität Ulm, 1995. English Edition: On positivity of discrete quadratic functionals.

[6] M. Bohner. Linear Hamiltonian difference systems: disconjugacy and Jacobi-type conditions. J. Math. Anal. Appl., 199(3):804-826, 1996. MR1386607(97a:39003)

[7] M. Bohner. Discrete Sturmian theory. Math. Inequal. Appl., 1(3):375-383, 1998. Preprint in Ulmer Seminare 1. MR1629392 (99e:39008)

[8] M. Bohner and O. Došlý. Disconjugacy and transformations for symplectic systems. Rocky Mountain J. Math., 27(3):707-743, 1997. MR1490271(99e:39007)

[9] M. Bohner, O. Došlý, and W. Kratz. An oscillation theorem for discrete eigenvalue problems. Rocky Mountain J. Math., 33(4):1233-1260, 2003. MR.2052485 (2005d:39083)

[10] M. Bohner, O. Došlý, and W. Kratz. Positive semidefiniteness of discrete quadratic functionals. Proc. Edinburgh Math. Soc., 46:627-636, 2003. MR2013957 (2004j:39022)

[11] O. Došlý and W. Kratz. A Sturmian separation theorem for symplectic difference systems. J. Math. Anal. Appl., 325:333-341, 2007. MR2273528 (2007j:39006)

[12] O. Došlý and W. Kratz. Oscillation theorems for symplectic difference systems. J. Difference Equ. Appl., 13(7):585-605, 2007. MR2336808

[13] J. Elyseeva. A transformation for symplectic systems and the definition of a focal point. Comput. Math. Appl., 47(1):123-134, 2004. MR2062731(2005b:39004)

[14] K. Feng. The Hamiltonian way for computing Hamiltonian dynamics. In Applied and Industrial Mathematics (Venice, 1989), volume 56 of Math. Appl., pages 17-35. Kluwer Acad. Publ., Dordrecht, 1991. MR.1147188 (92m:58043)

[15] R. Hilscher. Reid roundabout theorem for symplectic dynamic systems on time scales. Appl. Math. Optim., 43(2):129-146, 2001. MR.1814591(2002a:37118)

[16] R. Hilscher and V. Růžičková. Implicit Riccati equations and quadratic functionals for discrete symplectic systems. Int. J. Difference Equ., 1(1):135-154, 2006. MR2296502 
[17] W. Kratz. Quadratic Functionals in Variational Analysis and Control Theory, volume 6 of Mathematical Topics. Akademie Verlag, Berlin, 1995. MR1334092 (96f:34002)

[18] W. Kratz. Discrete oscillation. J. Difference Equ. Appl., 9(1):135-147, 2003. MR.1958308 (2004b:39020)

[19] J. Qi and S. Chen. Lower bound for the spectrum and the presence of pure point spectrum of a singular discrete Hamiltonian system. J. Math. Anal. Appl., 295(2):539-556, 2004. MR2072031 (2005e:47090)

[20] V. Růžičková. Discrete symplectic systems and definiteness of quadratic functionals. Ph.D. thesis, Masaryk University Brno, 2006.

[21] W. T. Reid. Oscillation criteria for self-adjoint differential systems. Trans. Amer. Math. Soc., 101:91-106, 1961. MR0133518 (24:A3349)

[22] W. T. Reid. Ordinary Differential Equations. John Wiley \& Sons, Inc., New York, 1971. MR0273082 (42:7963)

[23] W. T. Reid. Sturmian Theory for Ordinary Differential Equations. Springer-Verlag, New York, 1980. MR606199 (82f:34002)

[24] Y. Shi. Symplectic structure of discrete Hamiltonian systems. J. Math. Anal. Appl., 266(2):472-478, 2002. MR.1880519 (2002k:37093)

[25] Y. Shi. Spectral theory of discrete linear Hamiltonian systems. J. Math. Anal. Appl., 289(2):554-570, 2004. MR2026925 (2005a:39039)

[26] Y. Wang, Y. Shi, and G. Ren. Transformations for complex discrete linear Hamiltonian and symplectic systems. Bull. Aust. Math. Soc., 75(2):179-191, 2007. MR2312562 (2008c:39008)

Department of Mathematics and Statistics and Department of Economics and Finance, Missouri University of Science and Technology, Rolla, Missouri 65401

Department of Mathematics and Statistics, Masaryk University, CZ-61137, Brno, Czech Republic

Institut für Angewandte Analysis, Universität Ulm, D-89069 Ulm, Germany 